\title{
EVALUATION OF THE FOURTH MILLENNIUM DEVELOPMENT \\ GOAL REALISATION USING ROBUST AND NONPARAMETRIC TOOLS \\ OFFERED BY A DATA DEPTH CONCEPT
}

Ewa Kosiorowska, MD

General Practitioner, Internist Katowice, Poland

e-mail:e-mail:ewusia@mp.pl

Daniel Kosiorowski, Ph.D. Associate Professor

Cracow University of Economics

Department of Statistics

Rakowicka 27, 31-510 Cracow, Poland

e-mail:daniel.kosiorowski@uek.krakow.pl

Zygmunt Zawadzki, Ph.D. candidate

Cracow University of Economics

Master Studies

Rakowicka 27, 31-510 Cracow, Poland

e-mail: zawadzkizygmunt@gmail.com

Received 10 October 2014, Accepted 10 May 2015

\begin{abstract}
We briefly communicate the results of nonparametric and robust evaluation of the effects of the Fourth Millennium Development Goal of the United Nations. The main aim of the goal was reducing by two thirds, from 1990-2015, under five month's child mortality. Our novel analysis was conducted by means of very powerful and user friendly tools offered by the Data Depth Concept being a collection of multivariate techniques basing on multivariate generalizations of quintiles, ranges and order statistics. The results of our analysis are more convincing than the results obtained using classical statistical tools.
\end{abstract}

Keywords: The Fourth Millennium Goal; Data Depth Concept; Statistical Evaluation.

JEL Classification: C4, I3, I1. 


\section{Introduction}

The sample mean vector (MV) and the sample covariance matrix (COV) have been the standard estimators of location and scatter in multivariate statistics. They are working horses of the classical comparative analysis being a basis for a statistical evaluation of various socioeconomic goals. Unfortunately, economic data sets very often contain outliers or inliers of a various kind, which makes the MV and the COV useless due to their extreme sensitivity to atypical observations. Even one point departing from the main part of the data may destroy the estimation results obtained using them. In static, comparative Economics, we very often cannot use powerful tools of the classical multivariate statistics basing on the mean vector, the covariance matrix and the normality assumptions. In the dynamic Economy the situation is even more complicated.

This paper aims at presenting opportunities of conducting effective empirical research in a robust and nonparametric manner by means of not well known but very powerful and user friendly tools offered by the so called Data Depth Concept. The tools are implemented in our free R package DepthProc (see Kosiorowski, Zawadzki, 2014), which is available on CRAN servers. Our main motivations relate to our attempts of finding an empirical dimension of recent developments in the Theory of Cooperative Dynamic Games (CDG) (see Petrosjan, Yeung, 2012). Our interest in the CDG originates from our research program concerning the robust analysis of economic data streams. We have found that under certain circumstances a group of cooperating economic agents can be treated as a biological system processing data streams (see Kosiorowski, 2014).

Let us only recall that Robust Statistics aims at identifying a tendency represented by an influential majority of data and detecting observations departing from that tendency (see Marona et al., 2006). The main ideas of the robust statistics are closely tied with the notion of an influential majority of agents, investors, consumers - ruling the behaviour of a certain economic system. Unfortunately, methods of the robust statistics are rather rarely present in the current economic discussions, and in empirical or theoretical studies. The reasons for this situation are more complicated theory and the worse availability of statistical software in case of robust methods in comparison to the classical methods. The situation has changed recently however.

We understand the robustness of the estimator in terms of the influence function (IF) and the finite sample breakdown point (BP) - for further details see Maronna et. al. (2006). Let us recall that for a given distribution $F$ in $\mathbb{R}^{d}$ and an $\varepsilon>0$, the version of $F$ contaminated 
by an $\varepsilon$ amount of an arbitrary distribution $G$ in $\mathbb{R}^{d}$ is denoted by $F(\varepsilon, G)=(1-\varepsilon) F+\varepsilon G$. The influence function (IF) of an estimator $T$ at a given $\mathbf{x} \in \mathbb{R}^{d}$ for a given $F$ is defined

$$
I F(\mathbf{x} ; T, F)=\lim _{\varepsilon \rightarrow 0^{+}}\left(T\left(F\left(\varepsilon, \delta_{x}\right)\right)-T(F)\right) / \varepsilon
$$

where $\delta_{\mathbf{x}}$ is the point-mass probability measure at $\mathbf{x} \in \mathbb{R}^{d}$.

The $I F(\mathbf{x} ; T, F)$ describes the relative effect (influence) on $T$ of an infinitesimal point-mass contamination at $\mathbf{x}$, and measures the local robustness of $T$. An estimator with bounded IF (with respect to a given norm) is therefore robust (locally, as well as globally) and very desirable.

Let $\mathbf{X}^{n}=\left\{\mathbf{X}_{1}, \ldots, \mathbf{X}_{n}\right\}$ be a sample of size $n$ from $\mathbf{X}$ in $\mathbb{R}^{d}, d \geq 1$. The replacement breakdown point (BP) of a scatter estimator $V$ at $\mathbf{X}^{n}$ is defined as

$$
B P\left(V, \mathbf{X}^{n}\right)=\left\{\frac{m}{n}: \operatorname{tr}\left(V\left(X^{n}\right) V\left(X_{m}^{n}\right)^{-1}+V\left(X^{n}\right)^{-1} V\left(X_{m}^{n}\right)\right)=\infty\right\}
$$

where $\mathbf{X}_{m}^{n}$ is a contaminated sample resulting from replacing $m$ points of $\mathbf{X}^{n}$ with arbitrary values, $\operatorname{tr}()$ denotes trace of the matrix. The BP point serves as a measure of global robustness, while the IF function captures the local robustness of estimators.

In the context of empirical studies of the $\mathrm{CDG}$, we considered data sets related to the Millennium Declaration. Let us only briefly recall, that at the beginning of the century, world leaders come together at the United Nations and agreed on a general vision for the future through the Millennium Declaration. The Millennium Development Goals (MDGs) express the principles of human dignity, equality, and to free the world from extreme poverty (see UN Report, 2014). Although, the MDGs can be successfully evaluated for example within the SDEA (see Stone, 2002) framework - the success of these considerations crucially depends on a preliminary statistical analysis of the issue. In this work, we focus our attention on a statistical layer of the MDGs achievement evaluation.

For showing the usefulness of the Data Depth Concept (DDC) tools, let us consider an evaluation of the results of a program known as The Fourth Millennium Development Goal of the United Nations (4MDG). The main aim of the goal was reducing by two thirds, from 1990-2015, under five month's child mortality. Although, all MDGs are in fact only a general declaration of good-will, in the context of the 4MDG, the World Health Organization promotes four main strategies for obtaining the goal: 1. appropriate home care and timely treatment of complications for new-borns; 2. integrated management of childhood illness for all children 
under five years old; 3. expanded programme of immunisation; 4. infant and young child feeding. It should be stressed that in developing countries most deaths in the first year of life happen immediately after delivery or in the first days. Unfortunately medical care for new-born children is not available. Obstetric intervention protected from asphyxia, infections, treatment of childbirth complications is commonly neglected. One can improve infant mortality among children under five years old by the dissemination of vaccinations for childhood diseases, antibiotics for bacterial infections and oral rehydration therapy for diarrhoea (see Goldenberg, Jobe, 2001, and references therein). Taking on the concrete means by countries for obtaining the $4 \mathrm{MG}$ is beyond the direct control of the United Nations.

The rest of the paper is organized as follows: in Section 2, basic notions related to the Data Depth Concept and further used techniques are briefly described. In Section 3, the empirical analysis of the $4 \mathrm{MG}$ conducted by means of the depth tools is presented. The paper ends with some conclusions and references. All data sets and examples considered within the paper are available after installing the DepthProc $\mathrm{R}$ package.

\section{Selected data depth statistical tools}

\subsection{General notions of the DDC}

The Data depth concept (DDC) was originally introduced as a way to generalize the concepts of median and quantiles to the multivariate framework. A depth function $D(\cdot, F)$ associates with any $\mathbf{x} \in \mathbb{R}^{d}$ a measure $D(\mathbf{x}, F) \in[0,1]$ of its centrality with respect to (w.r.t.) a probability measure $F \in \mathcal{P}$ over $\mathbb{R}^{d}$ or w.r.t. an empirical measure $F_{n} \in \mathcal{P}$ calculated from a sample $\mathbf{X}^{n}$. The larger the depth (closer to one) of $\mathbf{x}$, the more central $\mathbf{x}$ is w.r.t. to $F$ or $F_{n}$. A point (or a set of points) for which a depth function attains a maximum is called a multivariate median induced by this depth. In the context of economic application of the DDC, it is worth focusing of our attention on the weighted $L^{p}$ depth. The weighted $L^{p}$ depth from a sample $\mathbf{X}^{n}=\left\{\mathbf{x}_{1}, \ldots, \mathbf{x}_{n}\right\} \subset \mathbb{R}^{d}$ is computed as

$$
D\left(\mathbf{x} ; \mathbf{X}^{n}\right)=\frac{1}{1+\frac{1}{n} \sum_{i=1}^{n} w\left(\left\|\mathbf{x}-\mathbf{X}_{i}\right\|_{p}\right)}
$$

where $w$ is appropriate non-decreasing and continuous on $[0, \infty)$ weight function, and $\|\cdot\|_{p}$ stands for the $L^{p}$ norm (when $p=2$ we have the usual Euclidean norm). 
The weighted $L^{p}$ depth function in a point, has the low breakdown point (BP) and unbounded influence function IF (the BP - expresses a minimal fraction of "bad" points in a sample making a procedure useless, the (IF) measures the sensitivity of a procedure on a point contamination for the BP and IF formal definitions). On the other hand, the weighted $L^{p}$ depth induced medians (multivariate location estimators) are globally robust with the highest BP for any reasonable estimator. The weighted $L^{p}$ medians are also locally robust with bounded influence functions for suitable weight functions. For the theoretical properties see Zuo (2004).

A symmetric projection depth $D(x, X)$ of a point $x \in \mathbb{R}^{d}, d \geq 1$ is defined as

$$
D(x, X)_{P R O}=\left[1+\sup _{\|u\|=1} \frac{\left|u^{T} x-\operatorname{Med}\left(u^{T} X\right)\right|}{\operatorname{MAD}\left(u^{T} X\right)}\right]^{-1}
$$

where Med denotes the univariate median, $\operatorname{MAD}(Z)=\operatorname{MED}(|Z-\operatorname{Med}(Z)|)$. Its sample version denoted by $D\left(x, X^{n}\right)$ or $D\left(x, F_{n}\right)$ is obtained by replacing population $F$ by its empirical counterpart $F_{n}$ calculated from the sample $X^{n}$. This depth is affine invariant and $D\left(x, F_{n}\right)$ converges uniformly and strongly to $D(x, F)$. The affine invariance ensures that our proposed inference methods are coordinate-free, and the convergence of $D\left(x, X^{n}\right)$ to $D(x, X)$ allows us to approximate $D(x, F)$ by $D\left(x, X^{n}\right)$ when $F$ is unknown. Induced by this depth, multivariate location and scatter estimators have very high breakdown points and bounded Hampel's influence function (for further details see Zuo, 2003). Procedures induced by $L^{p}$ depth are robust but not very robust in opposition to procedures induced by the projection depth.

The set of points for which depth takes a value not smaller than $\alpha \in[0,1]$ is a multivariate analogue of the quantile and is called the $\alpha$-central region,

$$
D_{\alpha}\left(\mathbf{X}^{n}\right)=\left\{\mathbf{x} \in \mathbb{R}^{d}: \underline{D}(\mathbf{x}, \mathbf{X}) \geq \alpha\right\}
$$

Every depth function induces a classification rule in a form: classify a point to a population in which the point has a maximal value. In an opposition to the density function, the depth function has a global nature i.e., e.g., that it expresses the centrality of a point w.r.t. a whole sample. This property is its disadvantage in the context of the classification of objects. A successful depth based classifier needs a local version of depth. A very successful concept of local depth was proposed in Paindaveine and Van Bever (2013). For a depth function $D(\cdot, P)$, the local depth with the locality parameter $\beta \in(0,1]$ and w.r.t. a point $x$ is the usual depth conditioned on a specially chosen neighbourhood of the point $x$. The neighbourhood covers $\beta$ fraction of the points concentrated around $x$. 
Next, very useful for the economic applications example of depth, is regression depth introduced in Rousseeuw and Hubert (1999). The deepest regression estimator is an example of robust regression. An up to date and detailed presentation of the DDC concept can be found in Mosler (2013). Below we only briefly present a multivariate generalisation of a well-known quantile-quantile plot, a generalization of the Wilcoxon rang sum statistic and the scale curve being a multivariate nonparametric scatter functional.

\subsection{Nonparametric and robust comparisons of multivariate samples}

The Depth vs. Depth plots (DD-plots)\}, introduced by Liu et al. (1999), is a user friendly two-dimensional graph allowing us a visual comparison of two samples of any dimension (multivariate quantile-quantile plot).

For two samples $\mathbf{X}^{n}=\left\{X_{1}, \ldots, X_{n}\right\}$ from $F$, and $\mathbf{Y}^{m}=\left\{Y_{1}, \ldots, Y_{m}\right\}$ from $G$, DD-plot is defined

$$
D D\left(F_{n}, G_{m}\right)=\left\{\left(D\left(\mathbf{z}, F_{n}\right), D\left(\mathbf{z}, G_{m}\right)\right), \mathbf{z} \in\left\{\mathbf{X}^{n} \cup \mathbf{Y}^{m}\right\}\right\}
$$

A difference in a location manifests in a form of a star-shaped pattern, whereas a difference in a scale manifests as a moon-shaped pattern.

It is easy to notice, that having two samples $\mathbf{X}^{n}$ and $\mathbf{Y}^{m}$ and using any depth function, we can compute depth values in a combined sample $\mathbf{Z}^{n+m}=\mathbf{X}^{n} \cup \mathbf{Y}^{m}$, assuming the empirical distribution calculated basing on all observations, or only on observations belonging to one of the samples $\mathbf{X}^{n}$ or $\mathbf{Y}^{m}$. If we observe $X_{l}$ 's depths are more likely to cluster tightly around the centre of the combined sample, while $Y_{l}$ s $Y_{l} s$ depths are more likely to scatter outlying positions, then we conclude $\mathbf{Y}^{m}$ was drawn from a distribution with larger scale.

The depth based multivariate Wilcoxon rang sum test is especially useful for the multivariate scale changes detection. For the samples $\mathbf{X}^{m}=\left\{\mathbf{X}_{1}, \ldots, \mathbf{X}_{m}\right\}, \mathbf{Y}^{n}=\left\{\mathbf{Y}_{1}, \ldots, \mathbf{Y}_{n}\right\}$, and a combined sample $\mathbf{Z}=\mathbf{X}^{n} \cup \mathbf{Y}^{m}$ the Wilcoxon statistic is defined as

$$
S=\sum_{i=1}^{m} R_{i}
$$

where $R_{i}$ denotes the rang of the $i$-th observation, $I=1, \ldots, m$ in the combined sample $R\left(\mathbf{x}_{l}\right)=\#\left\{\mathbf{z}_{j} \in \mathbf{Z}: D\left(\mathbf{z}_{j}, \mathbf{Z}\right) \leq D\left(\mathbf{x}_{l}, \mathbf{Z}\right)\right\}, l=1, \ldots, m$. The distribution of $S$ is symmetric about $E(S)=1 / 2 m(m+n+1)$, its variance is $D^{2}(S)=1 / 12 m n(m+n+1)$. For theoretical properties of the test see Li and Liu (2004) and Zuo and He (2006). 
For a sample depth function $D\left(x, Z^{n}\right), x \in \mathbb{R ^ { d }}, d \geq 2, Z^{n}=\left\{z_{1}, \ldots, z_{n}\right\} \subset \mathbb{R}^{d}, D_{\alpha}\left(Z^{n}\right)$ denoting $\alpha$-central region, we can define the scale curve

$$
S C(\alpha)=\left(\alpha, \operatorname{vol}\left(D_{\alpha}\left(Z^{n}\right)\right) \subset \mathbb{R}^{2}\right.
$$

for $\alpha \in[0,1]$.

The scale curve shows the expansion of a volume of $\alpha$-central region (the expansion around a multivariate median) in a relation to the outlyingness index (see Mosler, 2013, and references therein for further details).

Although the DDC is highly appreciated in the statistical literature, its popularity in the economic community seems to be relatively small. A reason for this relates to the absence of its tools in the popular commercial statistical software (except for the special case of a twodimensional boxplot - the bag plot) and high computational complexity of robust statistical techniques in general. Our R package DepthProc may significantly increase the popularity of the DDC among economists in the near future.

\section{Empirical Studies}

In our study we jointly considered following variables being officially recommended by the United Nations and having a medical justification (Goldberger, Jobe, 2001):

1. Children under 5 months mortality rate per 1,000 live births $\left(\mathrm{Y}_{1}\right)$.

2. Infant mortality rate ( $0-1$ year) per 1,000 live births $\left(\mathrm{Y}_{2}\right)$.

3. Children 1 year old immunized against measles, percentage $\left(\mathrm{Y}_{3}\right)$.

Data sets were obtained from http://mdgs.un.org/unsd/mdg/Data.aspx and are available within our free package DepthProc.

Figures 1-4 show weighted $L^{5}$ depth contourplots with the locality parameter $\beta=0.4$ for countries in 1990, 2000, 2005, 2010 considered w.r.t. variables $Y_{1}$ and $Y_{3}$, whereas Figures 5-8 present weighted $L^{5}$ depth contour plots with the locality parameter $\beta=0.4$ for countries in 1990, 2000, 2005, 2010 w.r.t. variables $Y_{2}$ and $Y_{3}$. Although we can notice a socio-economic development between 1990 and 2011 - the clusters of developed and developing countries are still evident in 2011 as they were in 1990. 


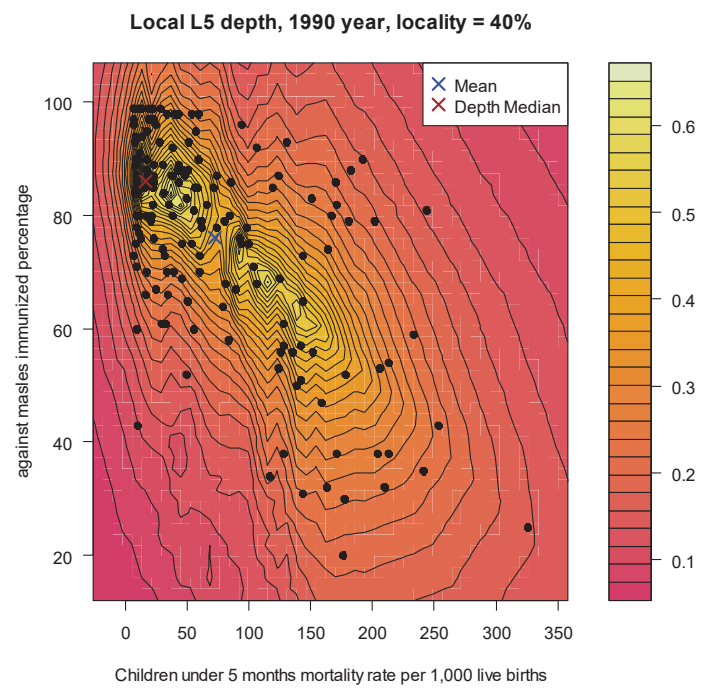

Fig. 1. The 4MG in 1990: $L^{5}$ depth contour plot $Y_{1}$ vs. $Y_{3}$, calculations DepthProc

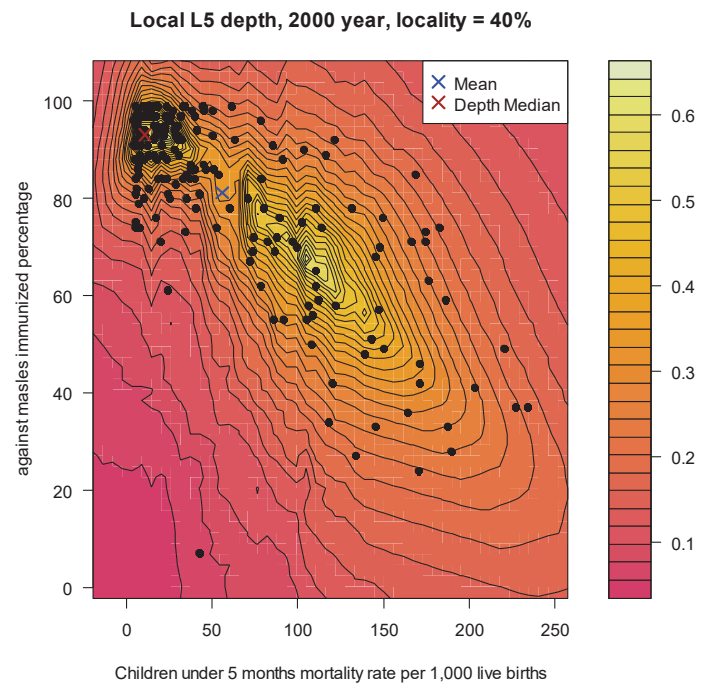

Fig. 2. The $4 \mathrm{MG}$ in 2000: $L^{5}$ depth contour plot $Y_{1}$ vs. $Y_{3}$, calculations DepthProc 


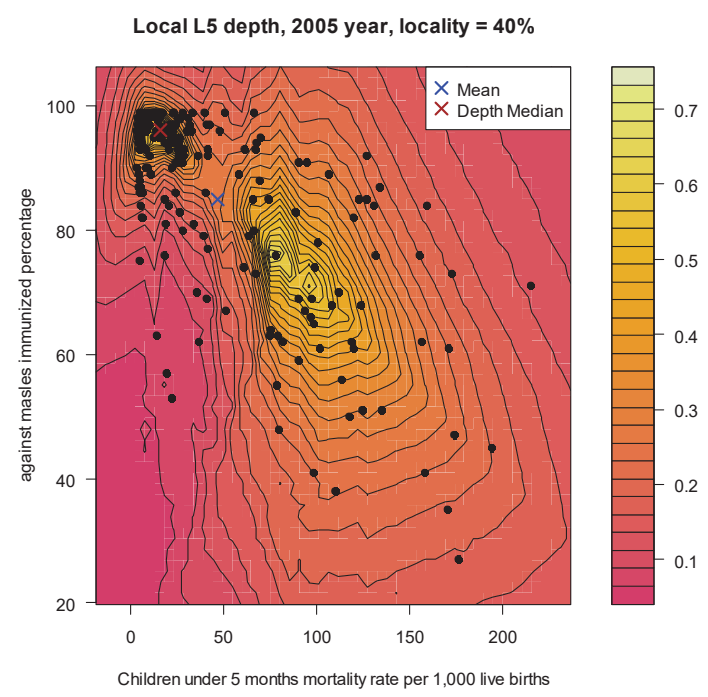

Fig. 3. The 4MG in 2005: $L^{5}$ depth contour plot $Y_{1}$ vs. $Y_{3}$, calculations DepthProc

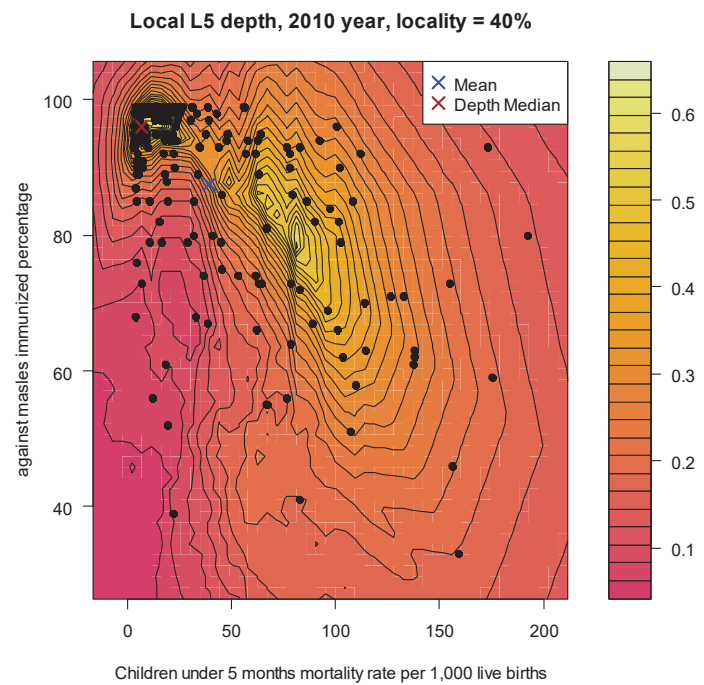

Fig. 4. The 4MG in 2010: $L^{5}$ depth contour plot $Y_{1}$ vs. $Y_{3}$, calculations DepthProc 


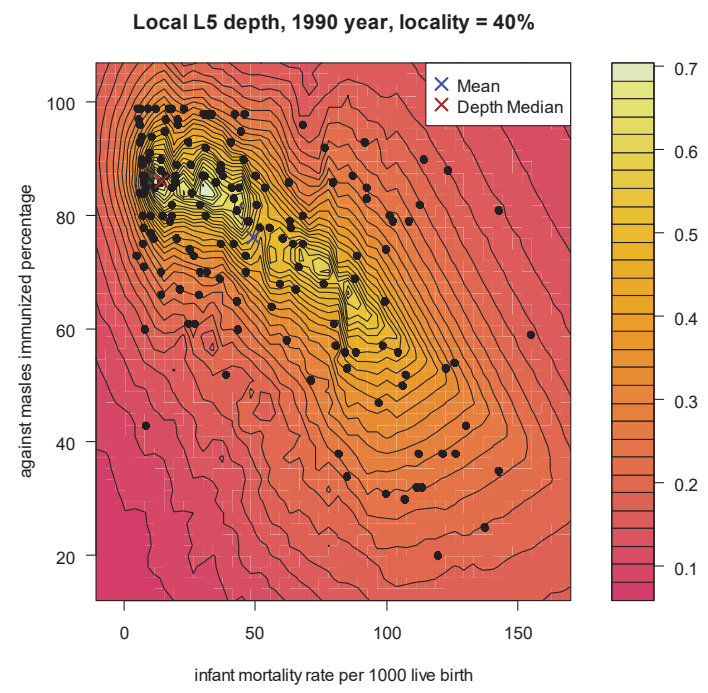

Fig. 5. The 4MG in 1990: $L^{5}$ depth contour plot $Y_{2}$ vs. $Y_{3}$, calculations DepthProc

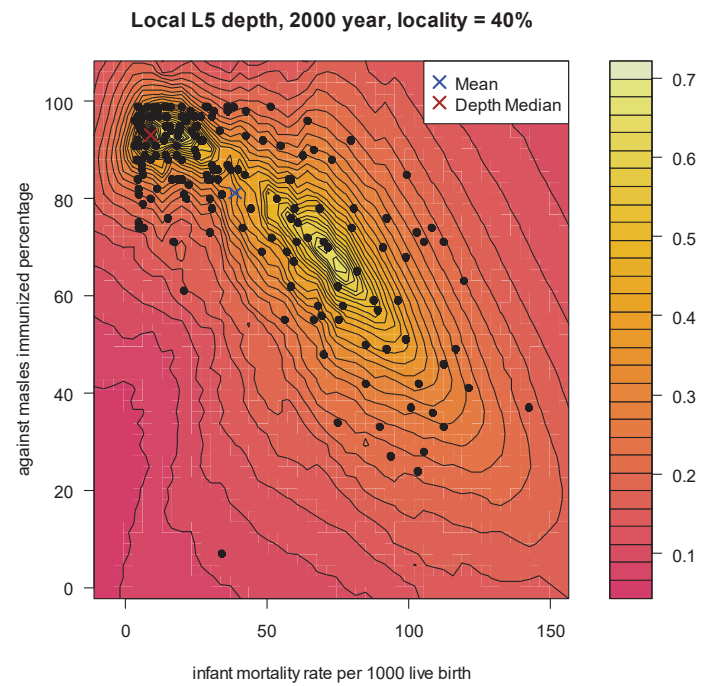

Fig. 6. The 4MG in 2000: $L^{5}$ depth contour plot $Y_{2}$ vs. $Y_{3}$, calculations DepthProc 


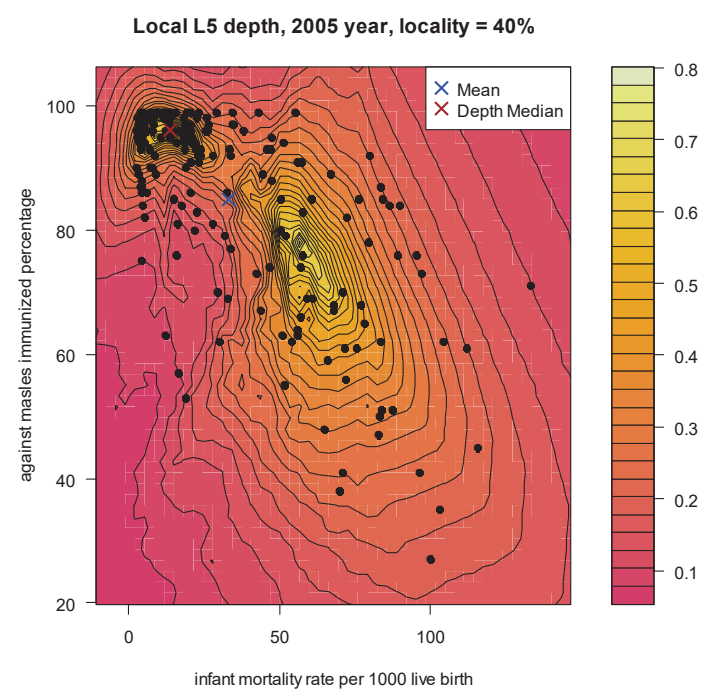

Fig. 7. The $4 \mathrm{MG}$ in 2005: $L^{5}$ depth contour plot $Y_{2}$ vs. $Y_{3}$, calculations DepthProc

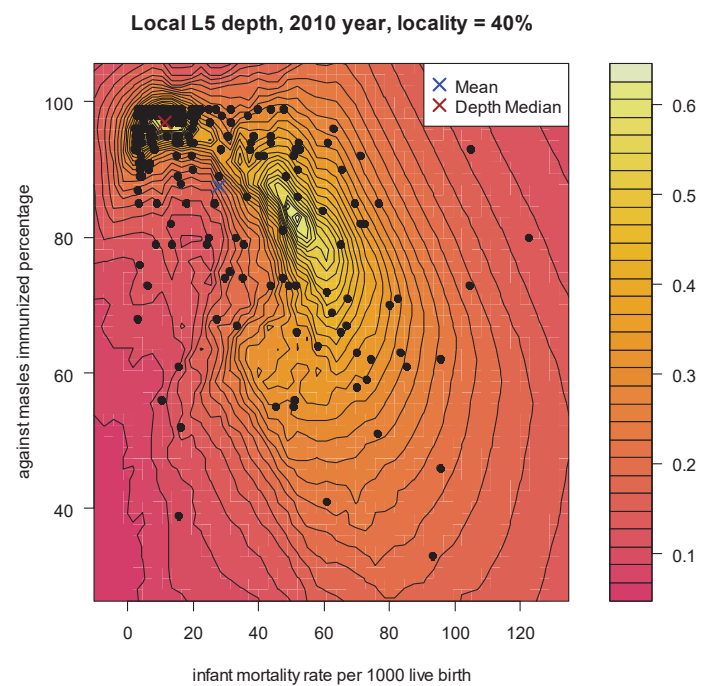

Fig. 8. The 4MG in 2010: $L^{5}$ depth contour plot $Y_{2}$ vs. $Y_{3}$, calculations DepthProc

For assessing changes in location of the centres and scatters of the data, between 1990 and 2011, we calculated $L^{1}$ medians (the robust measure of a centre) and $L^{5}$ weighted covariance matrices for $\left(\mathrm{Y}_{1}, \mathrm{Y}_{2}, \mathrm{Y}_{3}\right)$ (the robust measure of a scatter) which are presented in Table. 1 
It is easy to notice a significant development in the absolute values of the variables for a majority of the countries, both in the location as well as in the scale of the phenomenon. Figures 10 and 12. present DD-plots for inspecting location changes between 1990 and 2011 between 2000 and 2011 for countries considered as w.r.t. all variables $\left(\mathrm{Y}_{1}, \mathrm{Y}_{2}, \mathrm{Y}_{3}\right)$ and Figures 9 and 11 present DD-plots for inspecting scale changes for the same data. Patterns presented on these plots may be treated as evident arguments for positive changes between 1990 and 2011. Please note that the considered data suggest non-normality and the existence of outliers and hence the classical methods such as Hoteling tests for the samples comparison are not applicable here.

Table 1. Estimated location characteristics for $4 \mathrm{MG}$ indicators

\begin{tabular}{|c|c|c|c|c|c|c|c|c|c|}
\cline { 2 - 11 } \multicolumn{1}{c|}{} & \multicolumn{3}{c|}{$\mathrm{L}^{1}$ median } & \multicolumn{3}{c|}{ Projection median } & \multicolumn{3}{c|}{ Mean vector } \\
\hline Year & $\mathrm{Y}_{1}$ & $\mathrm{Y}_{2}$ & $\mathrm{Y}_{3}$ & $\mathrm{Y}_{1}$ & $\mathrm{Y}_{2}$ & $\mathrm{Y}_{3}$ & $\mathrm{Y}_{1}$ & $\mathrm{Y}_{2}$ & $\mathrm{Y}_{3}$ \\
\hline 1990 & 49.7 & 41.9 & 83.0 & 23.2 & 19.6 & 86.0 & 72.85 & 49.28 & 76.06 \\
\hline 1995 & 41.3 & 35.8 & 85.0 & 26.4 & 22.2 & 90.0 & 62.67 & 42.97 & 79.04 \\
\hline 2000 & 30.4 & 25.3 & 89.0 & 17.2 & 14.3 & 93.0 & 56.19 & 38.76 & 81.09 \\
\hline 2005 & 23.9 & 20.3 & 91.0 & 18.4 & 15.5 & 94.0 & 47.05 & 33.10 & 84.97 \\
\hline 2010 & 18.8 & 17.3 & 93.0 & 13.8 & 11.7 & 95.0 & 37.47 & 27.25 & 87.74 \\
\hline
\end{tabular}

Source: our own calculations, DepthProc package

$$
\begin{aligned}
C O V_{L^{5}}(1990) & =\left(\begin{array}{ccc}
2133 & 1295 & -397 \\
1295 & 817 & -240 \\
-397 & -240 & 264
\end{array}\right) ; C O V_{L^{5}}(2000)=\left(\begin{array}{ccc}
1449 & 910 & -384 \\
910 & 594 & -240 \\
-384 & -240 & 251
\end{array}\right) ; \\
C O V_{L^{5}}(2005) & =\left(\begin{array}{ccc}
1107 & 705 & -297 \\
705 & 468 & -192 \\
-297 & -192 & 218
\end{array}\right) ; C O V_{L^{5}}(2010)=\left(\begin{array}{ccc}
745 & 497 & -180 \\
497 & 342 & -121 \\
-180 & -121 & 175
\end{array}\right) .
\end{aligned}
$$

We performed induced by the $L^{2}$ depth multivariate Wilcoxon test for a scale change detection for the variables $\left(\mathrm{Y}_{1}, \mathrm{Y}_{2}, \mathrm{Y}_{3}\right)$ in 1990 and in 2011 and obtained: $\mathbf{W}=\mathbf{1 7 5 3 8}$ and p-value $=\mathbf{0 . 0 3 6 3}$. For the variables $\left(\mathrm{Y}_{1}, \mathrm{Y}_{3}\right)$ and using the projection depth we obtained $\mathrm{W}=15494.5, \mathrm{p}$-value $=0.7573$ and for the local Wilcoxon tests: beta $=0.8 \mathrm{~W}=12689.5$, $\mathrm{p}$-value $=0.001382$, beta $=0.7 \mathrm{~W}=11519.5, \mathrm{p}$-value $=1.065 \mathrm{e}-05$. We can conclude therefore, that both the scale and the location, changed in this period especially in the local dimension (local differences between countries changed. Figure 13. presents scale curves for the countries considered in the period 1990-2011 jointly w.r.t. all variables. The three-dimensional scatters 
around the three-dimensional $L^{2}$ medians decreased significantly in this period. Figures 14-16 present Student depth contour plots for the variables $Y_{1}, Y_{2}$ and $Y_{3}$ considered separately in 1990-2011. The Student depth relates to one dimensional location-scale problem, i.e., joint estimation of a location and scale parameters. The Student median is an effective and robust alternative for a mean and standard deviation. It indicates a value between the median and the mode. Its contour plot can be treated as a very powerful method of inspecting distributional assumptions. Further details can be found in Mizera and Müller (2004). Figures 14-16 show positive tendencies as to the changes of location and scale of variables together with retaining distributional properties - asymmetry related to the clusters of rich and poor countries remains nearly on the same level.

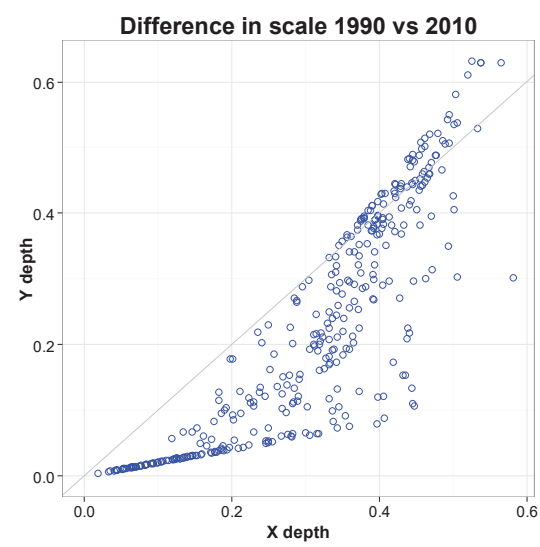

Fig. 9. DD plot for inspecting scale differences for $\left(Y_{1}, Y_{2}, Y_{3}\right)$ in 1990 and 2010.

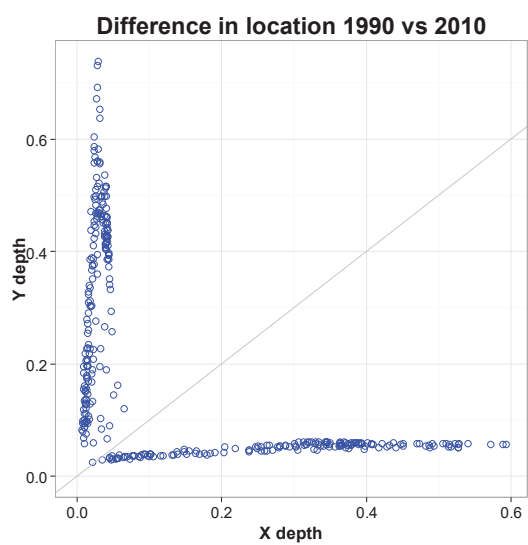

Fig. 10. DD plot for inspecting location differences for $\left(\mathrm{Y}_{1}, \mathrm{Y}_{2}, \mathrm{Y}_{3}\right)$ in 1990 and 2010. 


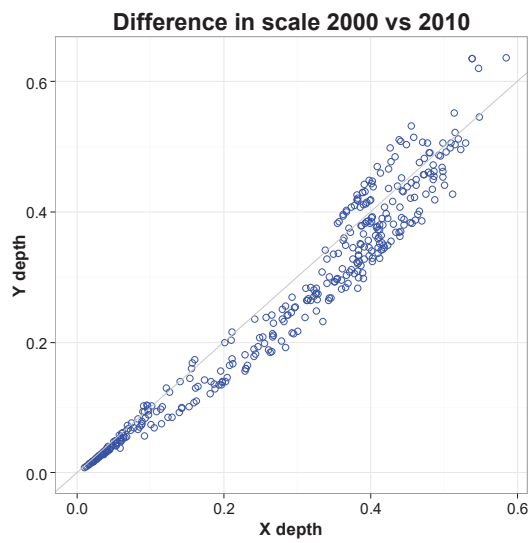

Fig. 11. DD plot for inspecting scale differences for $\left(\mathrm{Y}_{1}, \mathrm{Y}_{2}, \mathrm{Y}_{3}\right)$ in 2000 and 2010

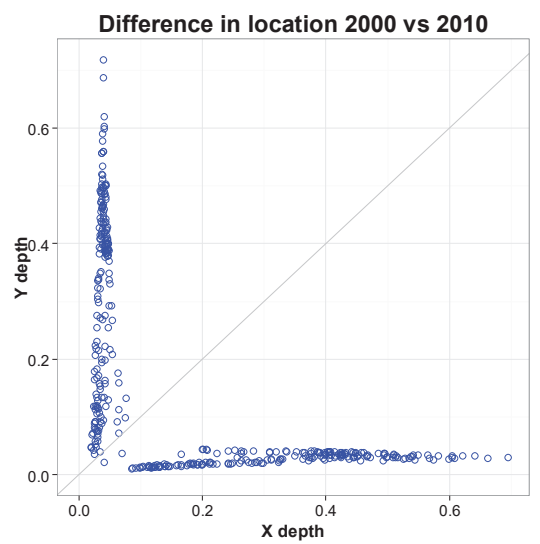

Fig. 12. DD plot for inspecting location differences for $\left(\mathrm{Y}_{1}, \mathrm{Y}_{2}, \mathrm{Y}_{3}\right)$ in 2000 and 2010

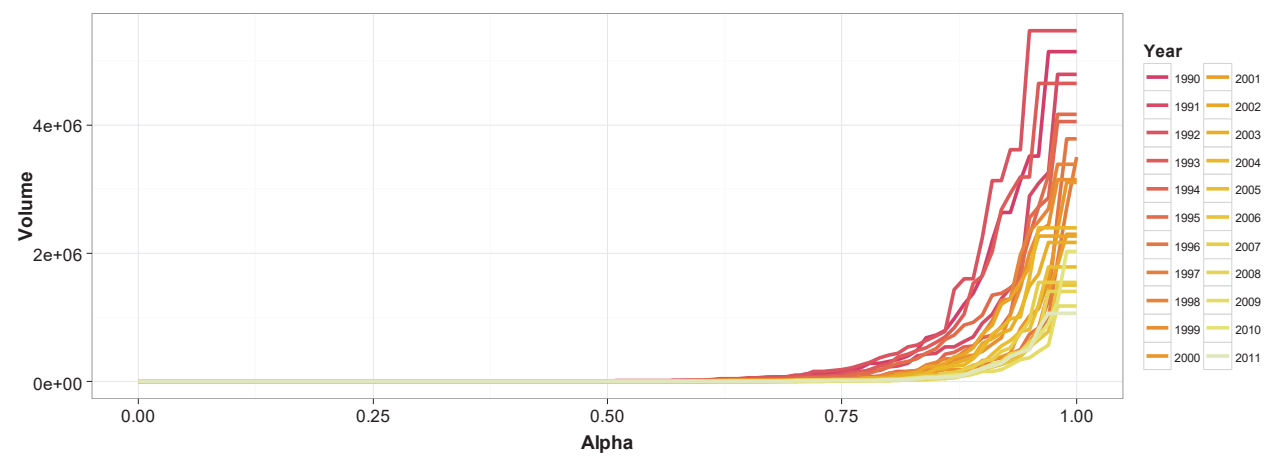

Fig. 13. Scale curves for $\left(\mathrm{Y}_{1}, \mathrm{Y}_{2}, \mathrm{Y}_{3}\right)$ 1990-2011, calculations DepthProc 


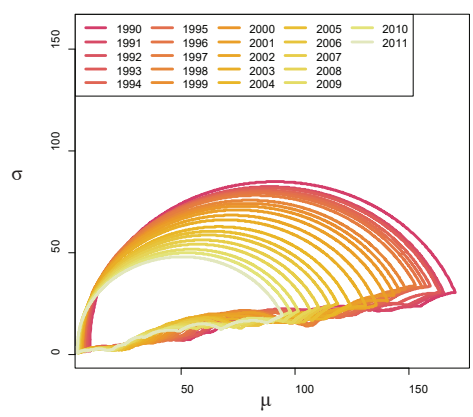

Fig. 14. Student depth contour plots $-Y_{1}$ in 1990-2011, calculations DepthProc

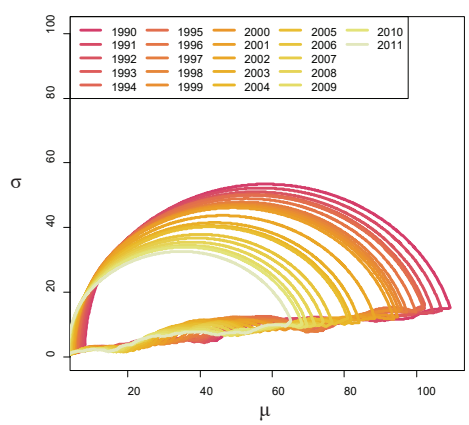

Fig. 15. Student depth contour plots $-Y_{2}$ in 1990-2011, calculations DepthProc

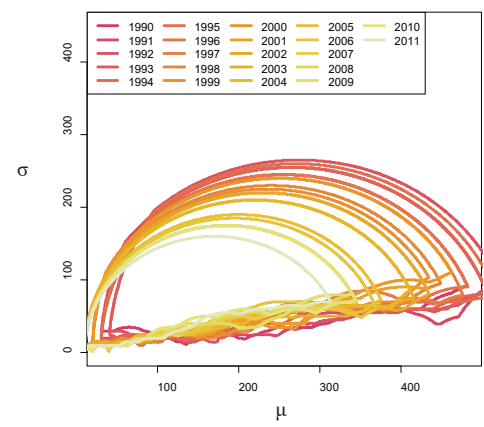

Fig. 16. Student depth contour plots $-Y_{3}$ in 1990-2011, calculations DepthProc 
Figure 17 presents a relation between variables $Y_{1}$ and $Y_{2}$ obtained by means of the simple deepest regression (DR - a robust method) and the popular least squares regression (LS a nonrobust method) in 1990. Figure 18 presents an analogous situation in 2011. Please note, that the strong relation between these variables is evident only when using the robust regression method (the slopes in 1990: LS - 0.28; DR -1.36, and the slopes in 2011: LS -0.34; DR -1.067).

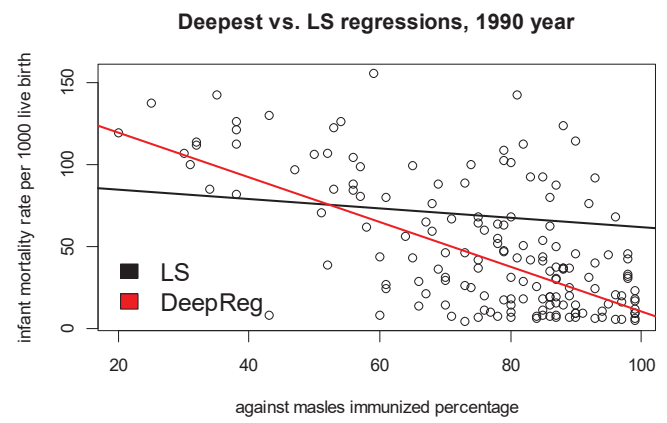

Fig. 17. Deepest vs. Least Squares regressions, $Y_{1}$ vs. $Y_{3}$ in 1990 , calculations DepthProc

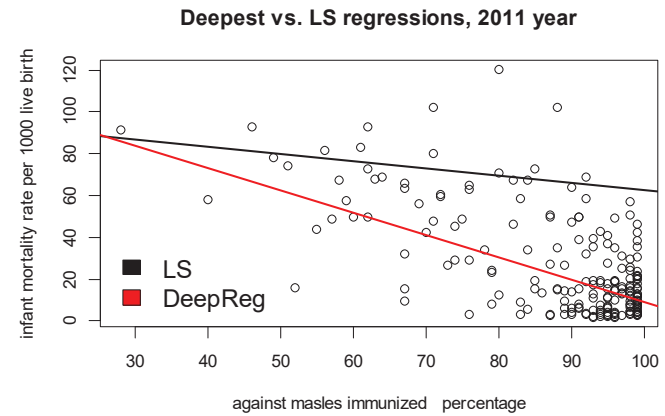

Fig. 18. Deepest vs. Least Squares regressions, $Y_{1}$ vs. $Y_{3}$ in 2011, calculations DepthProc

The results of the analysis lead us to the following conclusions:

1. There are big chances for obtaining the 4MG. In 2010 the decrease in under five month's child mortality was about $40 \%$ with robust estimates used.

2. For the variables considered jointly, both multivariate as well as univariate scatters decreased in 1990-2011. The decrease is especially evident in the closeness to the three-dimensional medians. 
3. Local differences between 1990 and 2011 are more evident than the global differences. The local tendencies among the countries indicate the positive direction of the changes in the health situation of children.

4. A comparison of the Student medians in 1990-2011 indicates significant onedimensional tendencies for obtaining the 4MDG. Asymmetries between rich and poor countries remain evident with a smaller overall dispersion however.

5. The estimated simple deepest regressions are arguments for the correct choice of the 4MDG goal realisation indicators. The relations between variables (interpreted as inputs and outputs) are more evident in comparison to relations indicated by the least squares regression. Efforts related to immunization have an evident impact on the infant mortality rate.

\section{Conclusions}

In the paper we presented a statistical evaluation of the 4MD conducted by means of robust and nonparametric tools offered by the DDC. The analysis was performed using data offered by the United Nations, which is a departure from normality and contains outliers. The results of the analysis look good for achieving the 4MD in the near future. Efforts related to immunization have an evident impact on the infant mortality rate. Values of the local multivariate Wilcoxon statistic show the positive direction of local changes within the considered countries between 1995 and 2011. A significant part of developing countries follow the tendency coined by developed countries.

Although achieving MDGs depends on a variety of qualitative and complex aspects like sound policies, good economic prospects, macroeconomic stability and peace - it is worth underlying that the results of the MDGSs realisation strongly depend on the statistical tools used for their evaluation. The robust multivariate methods offered by the depth concept underline the tendencies represented by the majority of countries. Using the local depth concept one can underline local tendencies among the countries, even when the data consists of inliers.

The differences between poor and rich countries still remains evident but an overall dispersion decreased in the considered period. The local depth contour plots show positive tendencies represented by developing countries. A distance between the centres of the clusters decreased in the considered period.

In the authors opinion, the DDC offers a comprehensive and user friendly family of robust and nonparametric statistical tools for the effects of various social programs evaluation 
purposes. These tools are implemented in the R package Depthproc and involve among others a multivariate quantile-quantile plot and multivariate global as well as local Wilcoxon tests and robust deepest regression, which should increase the DDC popularity in the economic community.

\section{Acknowledgement}

Daniel Kosiorowski thanks for the NCS financial DEC-011/03/B/HS4/01138 and CUE "Badnia statutowe 2015" supports.

\section{References}

Goldenberg, L.R., \& Jobe, A.H. (2001). Prospects for research in reproductive health and birth outcomes. JAMA, 285: 633-639.

Kosiorowski, D. \& Zawadzki, Z. (2014). DepthProc: An r package for robust exploration of multidimensional economic phenomena, http://arxiv.org/abs/1408.4542.

Li, J., \& Liu, R.Y. (2004). New nonparametric tests of multivariate locations and scales using data depth. Statistical Science, 19 (4): 686-696.

Liu, R., Parelius, J.M. \& Singh, K. (1999). Multivariate analysis by data depth: Descriptive statistics, graphics and inference (with discussion). The Annals of Statistics, 27: 783-858.

Maronna, R.A., Martin, R.D. \& Yohai, V.J (2006). Robust statistics - theory and methods. Chichester: John Wiley \& Sons.

Mizera, I. \& Müller, C.H (2004). Location-scale depth (with discussion). Journal of the American Statistical Association, 99: 949-966.

Mosler, K. (2013). Depth statistics. In: Robustness and Complex Data Structures, Festschrift in Honour of Ursula Gather. Ed. C. Becker, R. Fried, S. Kuhnt, 17-34. Springer Verlag.

United Nations Report. The millennium development goals report 2014, 2014, www.un.org/ millenniumgoals/2014.

Paindavaine, D. \& Van Bever, G. (2013). From depth to local depth: a focus on centrality. Journal of the American Statistical Association, 105: 1105-1119.

Petrosjan, L.A. \& Yeung, D.W.K. (2012). Subgame Consistent Economic Optimization - An Advanced Cooperative Dynamic Game Analysis. New York: Springer Verlag.

Rousseeuw, P.J. \& Hubert, M. (1999). Regression depth. Journal of the American Statistical Association, 94: 388-433. 
Stone, M. (2002). How not to measure the efficiency of public services (and how one might). Journal of Royal Statistical Society A, 3 (165): 405-434.

Zuo, Y. (2003). Projection Based Depth Functions and Associated Medians. The Annals of Statistics, 31 (5): 1460-1490.

Zuo, Y. (2004). Robustness of weighted $L^{p}-$ depth and $L^{p}$ median. Allgemaines Statistisches Archiv, 88: 215-234.

Zuo, Y. \& He, X. (2006). On the limiting distributions of multivariate depth based rank sum statistics and related tests. The Annals of Statistics, 34: 2879-2896. 